\title{
Variation in Crack Growth Properties and Its Effect on Fatigue Crack Growth Life for Al 7075-T6
}

\author{
${ }^{1}$ M. Faruk Yaren and ${ }^{* 1}$ A. O. Ayhan \\ ${ }^{1}$ Faculty of Engineering, Department of Mechanical Engineering Sakarya University, Turkey
}

\begin{abstract}
In this study, two different 3D crack propagation analyses are performed for Al 7075-T6. Each analysis has different crack growth constants (C-n) which are obtained by separate tests. The steps of crack growth analysis by using Fracture and Crack Propagation Analysis System (FCPAS) are detailed. As a result of crack growth analyses, stress intensity factor (SIF) distributions, growing crack fronts and crack growth lives are plotted and compared with the results of another study in the literature. The obtained results are in agreement with the literature data. Variation crack growth material properties and its effect on fatigue crack growth life is investigated. As a result, it has been shown that for the cases with the same stress intensity factor value, the variation crack growth material properties significantly change the crack growth life.
\end{abstract}

Key words: Crack Propagation, Paris-Erdogan Constants, Al 7075-T6, FCPAS, Crack Growth Life

\section{Introduction}

Mechanical behavior of materials can be determined experimentally. To deal with the uncertainties arising from material properties, environmental conditions and loadings, large number of tests are necessary to determine the mechanical properties of materials. Generally using average of results is simple, cheap, quick and acceptable for many applications. However, stochastic methods are more precise to reach sensitive results and are necessary to come up with more realistic life predictions.

Cracks can be seen in many engineering materials because of manufacturing defects, environment or operating conditions. From economic and safety aspects, correct life prediction accounting for uncertainties and variations is vital in aviation, space, defense and energy industries. Some crack growth models are proposed in literature [1-6] which are functions of some material constants; like crack growth parameter of Paris-Erdogan [1] and Forman [2], polynomial exponent of Walker [3], geometric shape factor of Wheeler [4]. These constants are produced from the crack growth curve. Therefore the variation in these constants causes difference in the life prediction of the part or structure. Due to the uncertainties listed above, estimating crack growth life with the help of stochastic theories is a frequently used method in recent studies.

Distribution of parameters and its effects on results are important in statistical studies. A large number of experiments are required to obtain distributions. There are some studies in literature which present data sets for crack growth rate [7-9]. Wu and $\mathrm{Ni}$ [7] conducted crack growth tests

*Corresponding author: Address: Faculty of Engineering, Department of Mechanical Engineering Sakarya University, 54187, Sakarya TURKEY. E-mail address: ayhan@ @akarya.edu.tr 
under the same conditions for 2024-T351 aluminum alloy. Different crack growth curves are obtained from their tests and it is seen that there is $\% 40$ difference between low and high values of predicted lives. Also results from Daniewicz and McDonald's experiments show nearly \%30 difference for 7075-T651 aluminum crack growth life [9]. These examples are sufficient to show the uncertainty and difficulty of crack growth life prediction.

Because of the loading history and load sequence effect, variable amplitude and random loadings applications involve challenges to estimate crack growth life. Overloading frequencies or magnitudes are the major factors for crack growth rate. Generally overloads are known to retard crack growth while underloads accelerate crack growth relative to the baseline crack growth rate. Some authors [10-12] proposed to use root mean square method to calculate effective stress ratio and crack growth life. Another approach to calculate crack growth life is through load by load interactions as retardation or acceleration, after classification of load interaction, crack growth rate is calculated using different equations or constants $[13,14]$.

In the presence of above variations, uncertainties and the resulting challenges, probabilistic fracture mechanics offer a good alternative for more accurate life estimation. In this study; fatigue crack growth rates for 7075-T651 aluminum are obtained by tests and Paris-Erdogan constants are determined for each test separately. From results, two sets of crack growth constants $(C-n)$ are chosen which yield maximum and minimum crack growth lives. A finite element model is prepared based on Daniewicz's tests [9]. Then crack growth analyses are done using C-n data sets which are obtained from the crack growth tests.

\section{Materials and Method}

In this study, crack growth lives are obtained from tests. Detailed information for tests are given in the next sub-section. A model is created and crack growth analyses are done using test data. Finite element model and essential information about crack growth analyses are also explained below.

\subsection{Experimental Study}

Compact tension specimens are machined from Al 7075-T651 rolled plate in L-T direction according to ASTM E647 [15]. Specimen thickness is $25 \mathrm{~mm}$. The length between center of loading hole and back of specimen are $50 \mathrm{~mm}$ which are labeled as ' $W$ ' in standard and other dimensions are its functions [15]. The experiments are performed on a $100 \mathrm{kN}-1100 \mathrm{~N} \cdot \mathrm{m}$ MTS axial-torsional fatigue test machine. Mode-I loading conditions are applied to specimen. $1.3 \mathrm{~mm}$ precrack is generated around notch before the start of the crack growth test. Back and front surface crack tips are recorded by two microscope cameras. Crack length is measured using a scale with half millimeter divisions pasted on the specimen. Also crack opening displacement gage is used to validate crack growth curves. After the test, crack lengths are measured as referenced pixel size by using camera records. As a result, crack growth rate $(d a / d N)$ versus alternating stress intensity factor $(\Delta K)$ graph is obtained. Using these graphs Paris-Erdogan constants ( $C$ and $n)$ are obtained. 
Table 1. Crack Growth Constants for Al 7075-T651

\begin{tabular}{lcc}
\hline & $\mathbf{C}$ & $\mathbf{n}$ \\
\hline Data Set 1 & $1.27 \times 10^{-7}$ & 3.161 \\
Data Set 2 & $2.06 \times 10^{-7}$ & 3.202 \\
\hline
\end{tabular}

\subsection{Fracture Analysis using FCPAS}

Fracture and Crack Propagation Analysis System, FCPAS [16] is a software, which employs enriched finite element formulation to solve linear elastic three-dimensional fracture mechanics problems. Further information about FCPAS and enriched elements can be found in previous studies. Three-dimensional fracture mechanics problems such as interfacial cracks [17], mixed mode fracture [18], functionally graded materials [19] and multiple cracks [20] have been solved accurately and efficiently using FCPAS.

In this study, a crack growth analysis is done for surface crack in a plate under cyclic loading. Dimensions of model is given in Fig.1. Due to the symmetry conditions in both horizontal and vertical directions, a quarter model is created and meshed in ANSYS ${ }^{\mathrm{TM}}$ [21]. Pressure is applied on the bottom surface and symmetry displacement conditions are identified right and top surfaces.

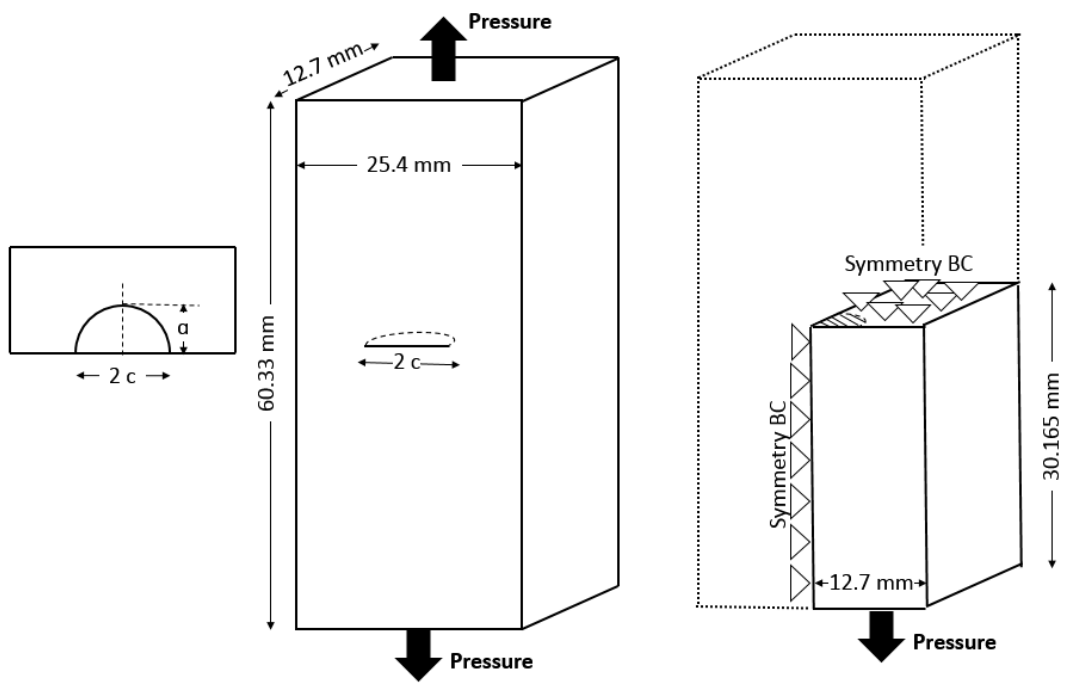

Figure 1. Details of the fracture model.

Initial crack depth " $a$ " is $1,27 \mathrm{~mm}$ and free surface length of the crack " $2 c$ " is $2,54 \mathrm{~mm}$. Pressure is $152 \mathrm{MPa}$ for stress ratio $\mathrm{R}=0,1$. All dimensions on model and the boundary conditions are the same as Ref [9]. Crack growth constants were obtained in experimental part of this study and they are given in Table 1. Lists of elements, nodes, pressure, and displacement are taken from ANSYS ${ }^{\mathrm{TM}}$ [21]. Using these lists a GEO file is created by a sub preprocessing problem in FCPAS. GEO file contains all information about the finite element model, such as loadings, nodes, element connectivity, crack definition and nodes around crack. Using GEO file FCPAS analyzes fracture 
problem and it gives stress intensity factors on crack front. If stress intensity factor is greater than threshold value of material, crack propagates till stress intensity factor is equal to critical stress intensity value. After each fracture analysis, stress intensity check process is done and if the crack propagates, all analyses steps listed previously are repeated. FCPAS process map and steps are given in Fig 2.

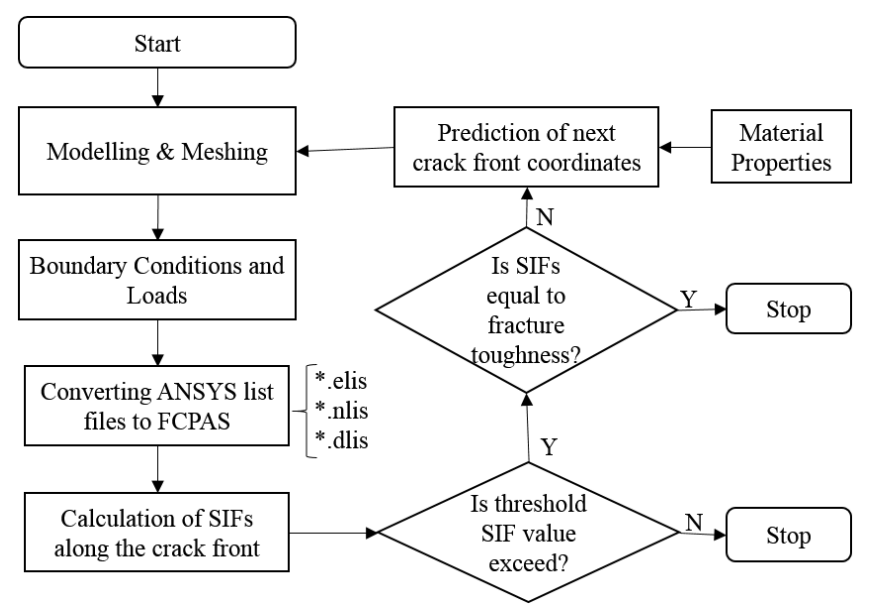

Figure 2. FCPAS Crack growth analysis procedure.

Crack propagation analysis is done incrementally by FCPAS. Accurate calculation of SIFs is important to predict the next crack front. As is known from the Paris-Erdogan equation [1], besides SIFs, material properties are also influential for crack propagation. Paris-Erdogan equation is given below as Eqn. 1.

$$
\frac{d a}{d N}=C(\Delta K)^{n}
$$

\section{Results}

In this section, results from the analyses are presented. Stress intensity factors and crack fronts are given. Crack growth life is compared with Ref [9].

\subsection{Fracture Analysis Results}

Crack growth analysis is done and crack fronts are predicted for two different Paris-Erdogan data sets. Two crack growth constant data sets are used which are given in Table 1. In Fig. 3, crack fronts which are obtained from two different crack growth analyses are presented. It can be seen in the Fig. 3 that initial crack geometry are the same for both. Aspect ratio of the last crack fronts are calculated as 0.81 at the end of two analyses. Aspect ratio in the experiments of the Ref [9] is also approximately 0.8 .

In Fig.4 stress intensity factors are given. $K_{l}$ stress intensity factor for first crack front is approximately $6 \mathrm{MPa} \cdot \mathrm{m}^{1 / 2}$. While crack propagates the stress intensity factor increases as expected. 
In the graph, 0.0 value for non-dimensional crack position indicates the mid-point of crack front. Accordingly, 1.0 means the free surface of crack. As seen in Fig. 4., $K_{1}$ SIF values at free surfaces are greater than those of mid-points, i.e., depth points. This means crack propagates faster on free surfaces than mid-point. Crack fronts in Fig. 3 confirms this requirement.

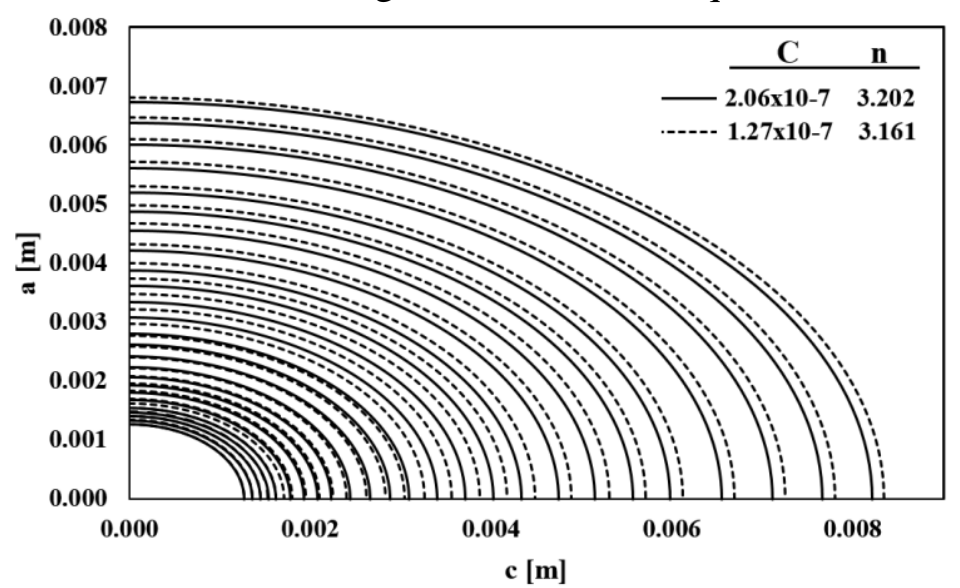

Figure 3. Crack fronts for two different Paris Erdogan constant data sets.

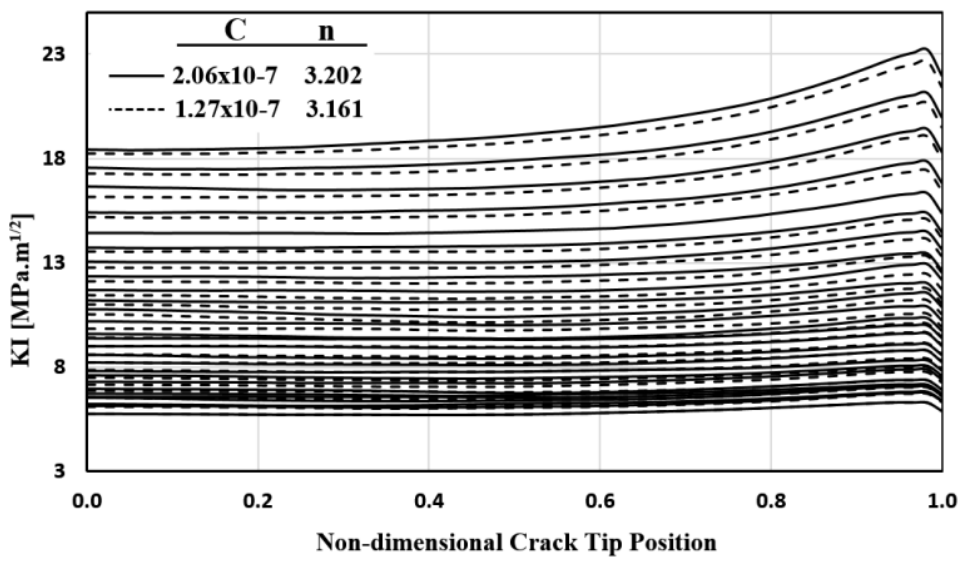

Figure 4. $K_{l}$ SIFs for two different Paris Erdogan constant data sets.

It can be seen in Fig 4. that for any crack front, SIFs are very close to each other for both data sets. Crack growth rate is a function of SIF. Since SIFs are equal, difference in crack growth life in Figs. 5 and 6 is because of the variation in crack growth properties. In Figs. 5 and 6, black markers represent FCPAS life predictions and the grey ones are from experiments by Daniewicz [9]. It can be seen on the graphs that, the life predictions are very close to the reference for the slowest crack growth rate. FCPAS life prediction by using crack growth constant data set 2, has shorter life than that of Ref. [9]. However, the difference between lower and upper bounds of life predictions from FCPAS and from Ref. [9] are also very close to each other. Thus, it can be concluded that the variations in $C$ and $n$ constants cause the observed scatter in crack propagation lives. 


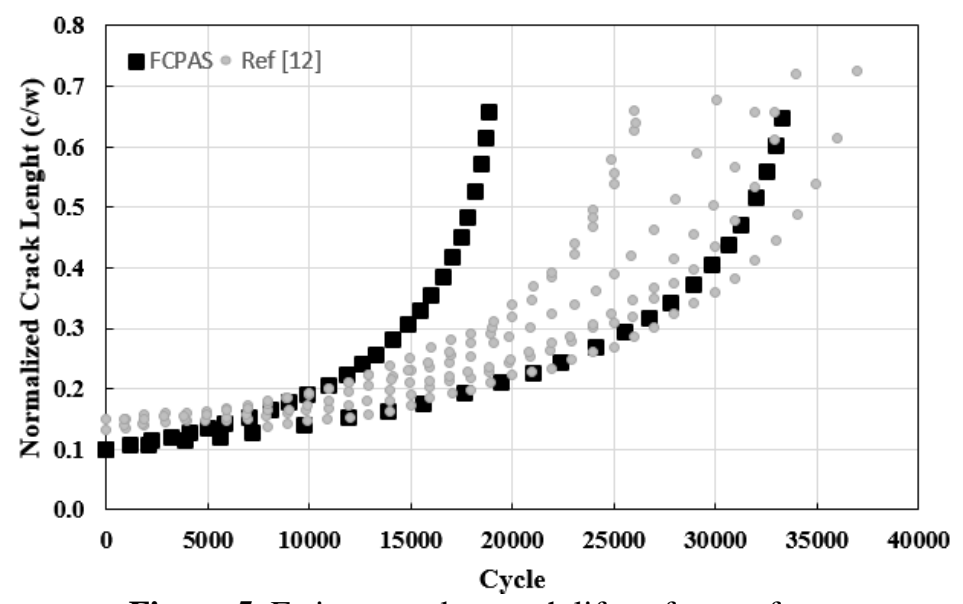

Figure 5. Fatigue crack growth life at free surface.

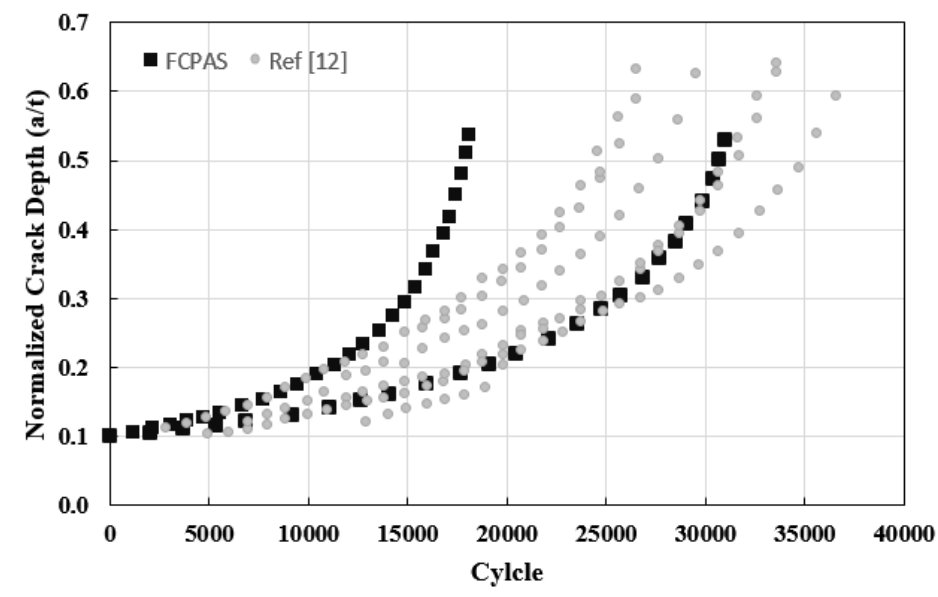

Figure 6. Fatigue crack growth life at the deepest point of crack.

\section{Conclusions}

Variation in crack growth life using Al 7075-T6 material properties is investigated in this paper. Fatigue crack growth tests are done and Paris-Erdogan crack growth constants are obtained from each test. A finite element model is created modelling the experiment in Ref [9] and crack propagation analyses were done using FCPAS and C-n constants, which are obtained from experimental part of this study. Predicted crack growth lives are compared with those of Ref [9] and results are in good agreement. Since, $n$ values are close to each other for high and low-crack growth rate properties, there is no difference in stress intensity factors between two analyses which include different crack growth constants. On the other hand, life predictions between two analyses have a great difference because the $\mathrm{C}$ values differ considerably between the two growth rate cases. As a future work, fatigue crack growth under variable amplitude loading is planned. Single over/under loading, block loading and their effects on crack growth parameters will be investigated. Using probabilistic methods, crack growth life estimation for more complicated cases will be studied. 


\section{Acknowledgements}

The financial support by The Scientific and Technological Research Council of Turkey (TUBITAK) under Project Number: 217M690 for this study is gratefully acknowledged.

\section{References}

[1] Paris, P., \& Erdogan, F. (1963). A critical analysis of crack propagation laws. Journal of basic engineering, 85(4), 528-533.

[2] Forman, R. G. (1972). Study of fatigue crack initiation from flaws using fracture mechanics theory. Engineering Fracture Mechanics, 4(2), 333-345.

[3] Walker, K. (1970). The effect of stress ratio during crack propagation and fatigue for 2024-T3 and 7075-T6 aluminum. In Effects of environment and complex load history on fatigue life. ASTM International.

[4] Wheeler, O. E. (1972). Spectrum loading and crack growth. Journal of basic engineering, 94(1), 181-186.

[5] Newman, J. C. (1981). A crack-closure model for predicting fatigue crack growth under aircraft spectrum loading. In Methods and models for predicting fatigue crack growth under random loading. ASTM International.

[6] McEvily, A. J., 1974. "Phenomenological and Microstructural Aspects of Fatigue". Presented at the Third International Conference on the Strength of Metals and Alloys, Cambridge,England; published by The Institute and The Iron and Steel Institutes, Publication, W36, PP.204-213.

[7] Wu, W. F., \& Ni, C. C. (2007). Statistical aspects of some fatigue crack growth data. Engineering Fracture Mechanics, 74(18), 2952-2963.

[8] Wei, R. P. (1970). Some aspects of environment-enhanced fatigue-crack growth. Engineering Fracture Mechanics, 1(4), 633-651.

[9] McDonald, V., \& Daniewicz, S. R. (2002). An experimental study of the growth of surface flaws under cyclic loading. In Fatigue and Fracture Mechanics: 32nd Volume. ASTM International.

[10]Hudson, C. M. "A root-mean-square approach for predicting fatigue crack growth under random loading." Methods and models for predicting fatigue crack growth under random loading. ASTM International, 1981.

[11]Kim, Sang Tae, Damir Tadjiev, and Hyun Tae Yang. "Fatigue life prediction under random loading conditions in 7475-T7351 aluminum alloy using the RMS model." International Journal of Damage Mechanics 15.1 (2006): 89-102.

[12] Manjunatha, C. M. "Fatigue Crack Growth Prediction under Spectrum Load Sequence in an Aluminum Alloy by K*-RMS Approach." International Journal of Damage Mechanics 17.6 (2008): 477-492.

[13] Johnson, W. S. "Multi-parameter yield zone model for predicting spectrum crack growth." Methods and models for predicting fatigue crack growth under random loading. ASTM International, 1981. 
[14]Dirik, Haydar, and Tuncay Yalçinkaya. "Fatigue crack growth under variable amplitude loading through XFEM." Procedia Structural Integrity 2 (2016): 3073-3080.

[15] Standard, A. S. T. M. "E647." Standard test method for measurement of fatigue crack growth rates. Annual book of ASTM Standards 3 (2000).

[16] Ayhan, A. O. "Simulation of three-dimensional fatigue crack propagation using enriched finite elements." Computers \& Structures 89.9 (2011): 801-812.

[17] Ayhan, A. O., and Herman F. Nied. "Finite element analysis of interface cracking in semiconductor packages." IEEE Transactions on Components and Packaging Technologies 22.4 (1999): 503-511.

[18] Ayhan, A. O. "Mixed mode stress intensity factors for deflected and inclined corner cracks in finite-thickness plates." International Journal of Fatigue 29.2 (2007): 305-317.

[19] Ayhan, A. O. "Stress intensity factors for three-dimensional cracks in functionally graded materials using enriched finite elements." International Journal of Solids and Structures 44.25 (2007): 8579-8599.

[20]Dündar, H., and Ayhan A. O. "Multiple and non-planar crack propagation analyses in thin structures using FCPAS." Frattura ed Integritá Strutturale 35 (2016): 360.

[21] ANSYS (2009) Theory Manual Version 12.0. Ansys Inc Canonsburg, PA, USA. 